\title{
Dynamics Simulation for an Upper-Limb Human-Exoskeleton Assistance System in a Latent-Space Controlled Tool Manipulation Task
}

\author{
Johannes Kühn, Tingli Hu, Moritz Schappler and Sami Haddadin ${ }^{1}$
}

\begin{abstract}
This paper introduces a complex dynamics simulation tool for an exoskeletal human upper limb assistant system. This heterogeneous simulation model couples the articulated dynamics of a six degrees-of-freedom (DoFs) wearable exoskeleton with an upper-limb human neuromechanics model of 12 skeletal and 42 muscular DoFs with corresponding neuromuscular controls. Furthermore, the process forces of tool-mediated manipulation tasks can be fed into the overall system. This simulation tool can be used for various purposes such as 1) design and evaluation of human-centered exoskeleton controllers 2) evaluating human motor control hypotheses during exoskeleton use and 3) investigating various properties and the performance on exoskeleton systems and manipulation tasks. Exemplary, this framework is used for designing and implementing a potential human kinematic latent-space trajectory controller for a power drilling manipulation task of an exoskeletal assistance.
\end{abstract}

\section{INTRODUCTION}

In the development of smart assistance systems for human upper limbs, this typically being exoskeleton-type devices, it becomes increasingly popular to consider insights from the human antetype into the overall design process. Especially, incorporating the human musculoskeletal system and control principles is expected to be beneficial from various perspectives. For example, coordinating the exoskeletal assistance system with the natural movement behavior of humans can be decisive for the success of such systems.

In order to design, test and build upper-limb exoskeletal assistance systems, a complete dynamics simulation of the overall system is required that consists of

- a human upper-limb musculoskeletal dynamics model combined with appropriate motor control law hypotheses in closed loop,

- an accurate exoskeleton dynamics and control model together with its coupling to the human user, and

- a manipulation task in which the human-exoskeleton system physically interacts with the environment.

Due to the complex dynamical behavior within each of the aforementioned subsystems and their interactions, a plausible and consistent dynamics simulation is a challenging task. Figure 1 shows a high-level overview of the involved components. Generally, the coupling between the human upper-limb musculoskeletal dynamics and an exoskeleton can be described by corresponding coupling dynamics. Both subsystems are controlled via a suitable motor control law. The interaction with the environment can be modeled as

${ }^{1}$ All authors are with Institut für Regelungstechnik, Fakultät für Elektrotechnik und Informatik, Gottfried Wilhelm Leibniz Universität Hannover, Appelstraße 11, D-30617 Hannover, GERMANY. Emails: \{kuehn;tingli.hu;schappler;haddadin@irt.uni-hannover.de\} a manipulation process acting on the endeffector of the exoskeletal system.

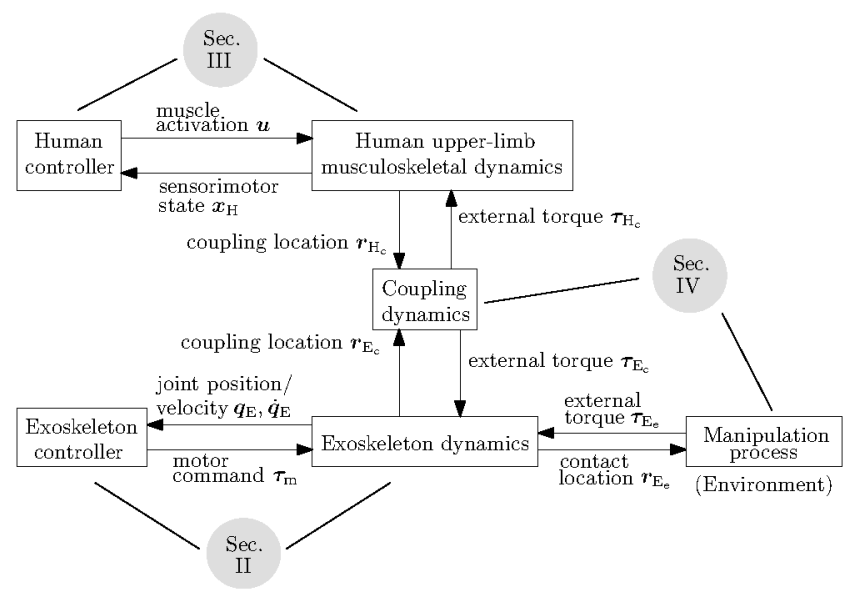

Fig. 1. Modeling a heterogeneous upper-limb human-exoskeleton system.

\section{A. State of the Art}

Robotics studies have addressed exoskeletons as assistance devices e.g. in the field of rehabilitation [1], [2]. Diverse exoskeleton control algorithms for supporting human users in object transport tasks exisit for upper [3] and lower limbs [4], [5].

Studies in the field of human neuromechanics focused on modeling the musculoskeletal plant dynamics including several motor control hypotheses [6], [7], [8], [9]. Those studies cover diverse body parts, e.g. legs [6], [7], neck [8], and upper limb [9] together with intransitive and toolmediated tasks.

The simplicity and effectiveness by which the central nervous system controls the upper limbs invoke a principle of dimensionality reduction [10]. A powerful framework aiming to account for it is the concept of sensorimotor synergies [11], [12]. The essential idea of the synergy framework is to represent control variables in a lower dimensional space, denoted the latent space in this paper, through which all mechanical degrees of freedom (DoFs) are simultaneously manipulated via suitable coupling behaviors [13]. Such latent spaces need to be identified on muscular [9] and kinematic level [11].

Considering this human synergistic behavior in exoskeleton control and design could improve such systems. So far, in the current state of the art (SotA) exoskeleton and human models are usually considered separately [3], [5], [6], 
This is the author's version of an article that has been published in the conference proceedings. Changes were made to this version by the publisher prior to publication

The final version of record is available at http://dx.doi.org/10.1109/SIMPAR.2018.8376286

TABLE I

State of the Art: Human-Exoskeleton Modeling, Control and Interaction

\begin{tabular}{|c|c|c|c|c|c|c|c|}
\hline \multirow[b]{2}{*}{ Literature } & \multirow[b]{2}{*}{ Body part } & \multicolumn{2}{|c|}{ Human Model } & \multicolumn{4}{|c|}{ Exoskeleton Model } \\
\hline & & \# Joints & \# Muscles & Control scheme & \# Joints & Control scheme & Task \\
\hline$[6],[7]$ & Legs & 8 & 30 & Static optimization & n.a. & n.a. & Cycling \\
\hline$[8]$ & Neck & 8 & 72 & PD + feedforward & n.a. & n.a. & Head movements \\
\hline [9] & $\begin{array}{l}\text { Shoulder- } \\
\text { elbow }\end{array}$ & 4 & 15 & $\begin{array}{l}\text { Muscular latent-space } \\
\text { PID control }\end{array}$ & n.a. & n.a. & Driving/steering \\
\hline [3] & n.a. & n.a. & n.a. & n.a. & 1 & $\begin{array}{l}\text { EMG-based force pre- } \\
\text { diction }\end{array}$ & $\begin{array}{l}\text { Loaded elbow } \\
\text { flexion/extension }\end{array}$ \\
\hline [5] & n.a. & n.a. & n.a. & n.a. & 1 & $\begin{array}{l}\text { Fuzzy impedance con- } \\
\text { trol }\end{array}$ & Leg swinging \\
\hline [14] & Legs & 12 & n.a. & n.a. & 12 & Fuzzy-PID/PID & $\begin{array}{l}\text { Walking, stair } \\
\text { ascent/descent, etc. }\end{array}$ \\
\hline [15] & Arm & Linearized & n.a. & n.a. & 4 & Impedance control & $\begin{array}{l}\text { Movements with no ex- } \\
\text { ternal force }\end{array}$ \\
\hline Our work & Shoulder-arm & 12 & 42 & $\begin{array}{l}\text { Kinematic latent-space } \\
\text { trajectory control }\end{array}$ & 6 & Gravity compensation & $\begin{array}{l}\text { Reaching movements/ } \\
\text { Power drilling }\end{array}$ \\
\hline
\end{tabular}

[7]. The few existing heterogeneous models often oversimplify the human dynamics. Typically, it is represented as a passive subsystem that e.g. smoothly follows exoskeleton movements [14], [15]. A significantly more human-centric dynamics simulation in the given context seems valuable.

Tab. I summarizes some of the most relevant existing works and highlights the contribution of this paper, which is detailed below.

\section{B. Contribution}

In this paper we introduce a simulation tool that combines wearable exoskeletal assistance systems with articulated human upper-limb musculoskeletal dynamics during tool use. It can be used for a wide range of system investigations and controller designs, such as

- design and evaluation of human-centered exoskeleton controllers, e.g., by considering human and exoskeleton posture, tracking error, muscle activation/effort or interaction forces,

- evaluation of human motor control hypotheses, and

- design and performance analysis of exoskeleton systems during tool-mediated manipulation tasks.

The main contributions are

- a dynamics simulation of human upper limb, exoskeleton and their respective coupling with each other via a virtual 6 DoF spring,

- kinematic synergy identification for a basic power drilling task,

- development of a human latent-space trajectory controller ${ }^{1}$ based on the identified synergies, and

- kinematic and muscular analysis of the latent-space trajectory controlled system.

${ }^{1}$ Note that this control hypothesis is only for evaluating our framework. Its validity is not of interest in this paper.
Specifically, we discuss the recently developed 6 DoF exoskeleton system $3 \mathrm{rd}$ arm. It was designed to support craftsmen by compensating the weight of power tools, thus relieving the human musculoskeletal system from large payloads. The human upper-limb neuromechanics model consists of 12 skeletal and 42 muscular DoFs. Based on the identification of kinematic synergies for an exemplary power drilling manipulation task, we introduce and analyze a novel human latent-space trajectory controller.

The remainder of the paper is organized as follows (see also Fig. 1). Section II introduces the general dynamics model of an upper-limb exoskeleton, the model of the 3rd arm, and its controller. Section III provides the human shoulder-arm neuromechanics model. Furthermore, the synergy identification approach and the human latent-space trajectory control law are explained. The coupling between human and exoskeleton during a simple drilling process model is outlined in Sec. IV. Finally, in Sec. V quantitative results for two cycles of a human-driven power drilling manipulation task are discussed. Section VI concludes the work.

\section{EXOSKELETON SIMULATION MODEL}

\section{A. Rigid-body dynamics}

The behavior of an $n$ DoF upper-limb exoskeleton system interacting with a human craftsman and a tool-mediated manipulation process acting on the endeffector can be modeled as

$$
\begin{array}{r}
\boldsymbol{M}_{\mathrm{E}}\left(\boldsymbol{q}_{\mathrm{E}}\right) \ddot{\boldsymbol{q}}_{\mathrm{E}}+\boldsymbol{c}_{\mathrm{E}}\left(\boldsymbol{q}_{\mathrm{E}}, \dot{\boldsymbol{q}}_{\mathrm{E}}\right)+\boldsymbol{g}_{\mathrm{E}}\left(\boldsymbol{q}_{\mathrm{E}}\right)+\boldsymbol{h}_{\mathrm{E}}\left(\boldsymbol{q}_{\mathrm{E}}\right)= \\
\boldsymbol{\tau}_{\mathrm{m}}+\boldsymbol{J}_{\mathrm{E}_{\mathrm{c}}}^{\mathrm{T}}\left(\boldsymbol{q}_{\mathrm{E}}\right) \mathcal{F}_{\mathrm{E}_{\mathrm{c}}}+\boldsymbol{J}_{\mathrm{E}_{\mathrm{e}}}^{\mathrm{T}}\left(\boldsymbol{q}_{\mathrm{E}}\right) \mathcal{F}_{\mathrm{E}_{\mathrm{e}}},
\end{array}
$$

where $\boldsymbol{M}_{\mathrm{E}} \in \mathbb{R}^{n \times n}, \boldsymbol{c}_{\mathrm{E}} \in \mathbb{R}^{n}$ and $\boldsymbol{g}_{\mathrm{E}} \in \mathbb{R}^{n}$ are the inertia matrix, Coriolis/centrifugal and gravitational terms, respectively. The term $\boldsymbol{h}_{\mathrm{E}} \in \mathbb{R}^{n}$ contains other nonlinear effects such as elastic joint limits [16] or supportive mechanical spring elements, while $\tau_{\mathrm{m}} \in \mathbb{R}^{n}$ denotes the motor torques. 
The external wrench $\mathcal{F}_{\mathrm{E}_{\mathrm{c}}} \in \mathbb{R}^{6}$ caused by the human user acts at the coupling location $E_{c}$ and is included via the corresponding Jacobian $\boldsymbol{J}_{\mathrm{E}_{\mathrm{c}}} \in \mathbb{R}^{6 \times n}$. The external wrench $\mathcal{F}_{\mathrm{E}_{\mathrm{e}}} \in \mathbb{R}^{6}$ caused by manipulation processes acts at the endeffector and is projected to joint space via the Jacobian $\boldsymbol{J}_{\mathrm{E}_{\mathrm{e}}} \in \mathbb{R}^{6 \times n}$. We distinguish between $E_{e}$ and $E_{c}$ in order to take into account the true locations of force application. However, note that it would be possible to relate both forces to only one point for the current model at hand. Jacobians and wrenches are expressed in the inertial frame. Note also that the joint position vector $\boldsymbol{q}_{\mathrm{E}} \in \mathbb{R}^{n}$ denotes the system minimal coordinates after eliminating kinematic constraints that may occur due to closed kinematic loops and gear rolling motions.

\section{B. The 3rd Arm}

In this paper, the exoskeleton model from the $3 \mathrm{rd} \mathrm{Arm}$ project $^{2}$ is used. This system aims to support a healthy human craftsman by holding the weight of a power tool, e.g. a drilling machine, see Fig. 2.

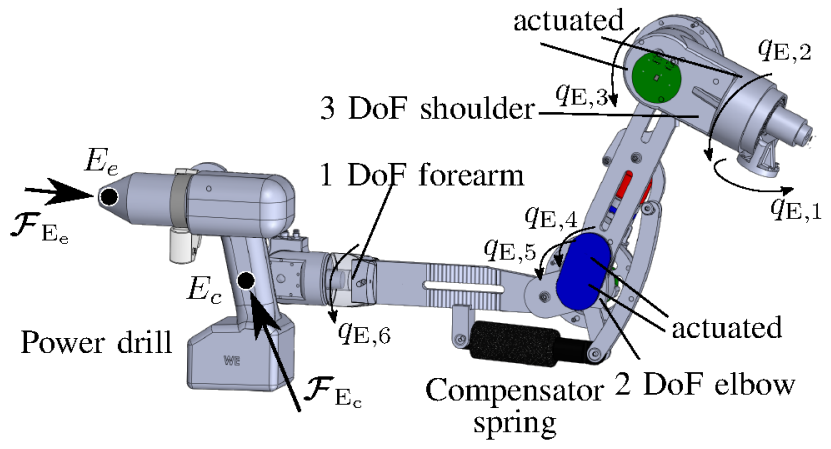

Fig. 2. CAD model of the $3 r d$ Arm equipped with a power drill. $\mathcal{F}_{\mathrm{E}_{e}}$ and $\mathcal{F}_{\mathrm{E}_{e}}$ denote the external wrenches interacting with the exoskeleton via endeffector $E_{e}$ and handle coupling location $E_{c}$.

The main structure is composed of three shoulder joints $q_{\mathrm{E}, 1-3}$, two elbow joints $q_{\mathrm{E}, 4-5}$ following the anatomy of the human elbow, and one forearm rotation joint $q_{\mathrm{E}, 6}$, i.e., $n=6$ DoFs. The joints $q_{\mathrm{E}, 2,3,4,5}$ are motor-driven, whereas $q_{\mathrm{E}, 1,6}$ are back-drivable. An additional kinematic parallel mechanism is able to couple the shoulder joint $q_{\mathrm{E}, 3}$ (responsible for flexion/extension) with the elbow joints $q_{\mathrm{E}, 4,5}$. This parallel mechanism allows to actuate these joints with the same motor, and leads to synergy-like motions during flexions/extensions of the human arm. In principle this would reduce the total number of DoFs by one. However, in this paper this kinematic coupling has no effect on the demonstrated power drilling task, thus $n=6$ still holds. Furthermore, a built-in spring supports gravity compensation of the exoskeleton and the power drill mounted on the endeffector.

An "X"-shaped backrest frame is used to absorb and transfer the load into the back and hip. The well known

\footnotetext{
2"3rd Arm" - Craftsmen-Force-Assistance with adaptive Human-Machine Interaction, see https://www.irt.uni-hannover.de/36.html. Founded by German Federal Ministry of Education and Research. The overall mechanism of the $3 r d \mathrm{Arm}$ is courtesy of M. Winkler and the $3 \mathrm{rd} \mathrm{Arm}$ project.
}

problem of asymmetric loading, caused by the dead weight, is to be partially eliminated by positioning the battery and electronics on the other side of the exoskeleton. However, this problem was not completely solved in the project and is thus not analyzed in this paper.

The specific dynamics of the $3 r d$ Arm follow (1), where $h_{\mathrm{E}}:=h_{\mathrm{E}}^{\prime}+\tau_{\mathrm{s}}$ and $\tau_{\mathrm{s}} \in \mathbb{R}^{6}$ originates from the built-in spring to counteract gravity effects, see Fig. $2 . h_{\mathrm{E}}^{\prime}$ contains other nonlinear effects.

\section{Exoskeleton Control}

In this paper, the exoskeleton controller provides a active gravity compensation. It compensates for difference of $\boldsymbol{g}_{\mathrm{E}}\left(\boldsymbol{q}_{\mathrm{E}}\right)$ and $\boldsymbol{\tau}_{\mathrm{s}}\left(\boldsymbol{q}_{\mathrm{E}}\right)$ by

$$
\tau_{\mathrm{m}}=\boldsymbol{S}\left[\hat{\boldsymbol{\tau}}_{\mathrm{s}}\left(\boldsymbol{q}_{\mathrm{E}}\right)+\hat{\boldsymbol{g}}_{\mathrm{E}}\left(\boldsymbol{q}_{\mathrm{E}}\right)\right],
$$

where the accent $\hat{\square}$ indicates estimations of the corresponding terms. The selection matrix $\boldsymbol{S}=\operatorname{diag}\left(\left[\begin{array}{llllll}0 & 1 & 1 & 1 & 1\end{array}\right]^{\mathrm{T}}\right)$ ensures that only motor-driven joints are taken into consideration.

\section{HUMAN UPPER-LIMB SIMULATION MODEL}

\section{A. Upper-limb musculoskeletal dynamics}

The $j$-skeletal DoF upper-limb musculoskeletal dynamics model interacting with an exoskeleton is essentially a multi-body dynamics system driven by its nonlinear $k$-DoF muscular actuators. Similar to (1) it can be written as

$$
\begin{gathered}
\boldsymbol{M}_{\mathrm{H}}\left(\boldsymbol{q}_{\mathrm{H}}\right) \ddot{\boldsymbol{q}}_{\mathrm{H}}+\boldsymbol{c}_{\mathrm{H}}\left(\boldsymbol{q}_{\mathrm{H}}, \dot{\boldsymbol{q}}_{\mathrm{H}}\right)+\boldsymbol{g}_{\mathrm{H}}\left(\boldsymbol{q}_{\mathrm{H}}\right)+\boldsymbol{h}_{\mathrm{H}}\left(\boldsymbol{q}_{\mathrm{H}}\right)= \\
\boldsymbol{J}_{\mathrm{M}}^{\mathrm{T}}\left(\boldsymbol{q}_{\mathrm{H}}\right) \boldsymbol{F}_{\mathrm{M}}+\boldsymbol{J}_{\mathrm{H}_{\mathrm{c}}}^{\mathrm{T}}\left(\boldsymbol{q}_{\mathrm{H}}\right) \mathcal{F}_{\mathrm{H}_{\mathrm{c}}}, \\
\boldsymbol{F}_{\mathrm{M}}=\boldsymbol{f}\left(\boldsymbol{u}, \boldsymbol{l}_{\mathrm{M}}\left(\boldsymbol{q}_{\mathrm{H}}\right), \dot{\boldsymbol{l}}_{\mathrm{M}}\left(\boldsymbol{q}_{\mathrm{H}}, \dot{\boldsymbol{q}}_{\mathrm{H}}\right)\right),
\end{gathered}
$$

where $\boldsymbol{q}_{\mathrm{H}}, \dot{q}_{\mathrm{H}}, \ddot{q}_{\mathrm{H}} \in \mathbb{R}^{j}$ denote the joint-space position, velocity, and acceleration, respectively. $M_{\mathrm{H}} \in \mathbb{R}^{j \times j}$ is the positive-definite, symmetric joint-space inertia matrix, $\boldsymbol{c}_{\mathrm{H}} \in$ $\mathbb{R}^{j}$ the joint-space Coriolis and centrifugal force, $g_{\mathrm{H}} \in \mathbb{R}^{j}$ the joint-space gravity, and $\boldsymbol{h}_{\mathrm{H}} \in \mathbb{R}^{j}$ includes other nonlinear forces. The Jacobian $J_{\mathrm{H}_{\mathrm{c}}} \in \mathbb{R}^{6 \times j}$ to the coupling point $H_{c}$ incorporates the external wrench $\mathcal{F}_{\mathrm{H}_{\mathrm{c}}} \in \mathbb{R}^{6}$, caused by the exoskeleton and its interaction with the manipulation process. The stacked muscle-space force vector $\boldsymbol{F}_{\mathrm{M}} \in \mathbb{R}_{\geqslant 0}^{k}$ is exerted by musculotendinous units which are modeled as Hill-type muscles $\boldsymbol{f}(\cdot)$ in relation with muscle activation $\boldsymbol{u} \in[0,1]^{k}$, musculotendon length and its change rate $l_{\mathrm{M}}, \dot{l}_{\mathrm{M}} \in \mathbb{R}^{k}$ [17]. The muscle-skeletal Jacobian matrix $\boldsymbol{J}_{\mathrm{M}} \in \mathbb{R}^{k \times j}$ is defined as

$$
\boldsymbol{J}_{\mathrm{M}}\left(\boldsymbol{q}_{\mathrm{H}}\right)=-\frac{\partial \boldsymbol{l}_{\mathrm{M}}\left(\boldsymbol{q}_{\mathrm{H}}\right)}{\partial \boldsymbol{q}_{\mathrm{H}}}
$$

where the negative sign originates from the natural opposition between $\boldsymbol{F}_{\mathrm{M}}$ and $\boldsymbol{l}_{\mathrm{M}}$, i.e., muscle contraction (positive force) tends to shorten the muscle length (negative length change). The human motor controller generates an appropriate muscle activation $\boldsymbol{u}$. 


\section{This is the author's version of an article that has been published in the conference proceedings. \\ Changes were made to this version by the publisher prior to publication. \\ The final version of record is available at http://dx.doi.org/10.1109/SIMPAR.2018.8376286}

In this work, an upper-limb musculoskeletal model composed of $j=12$ skeletal DoFs and $k=42$ muscles/subregions was developed. The skeletal DoFs are associated to the anatomical joints sternoclavicular, acromioclavicular, glenohumeral, humeroulnar, radioulnar, and radiocarpal. The scapulothoracic sliding plane is considered in $\boldsymbol{h}_{\mathrm{H}}$, see (3). Fig. 3 shows the current visualization rendered in Unity (Unity Technologies SF, USA).
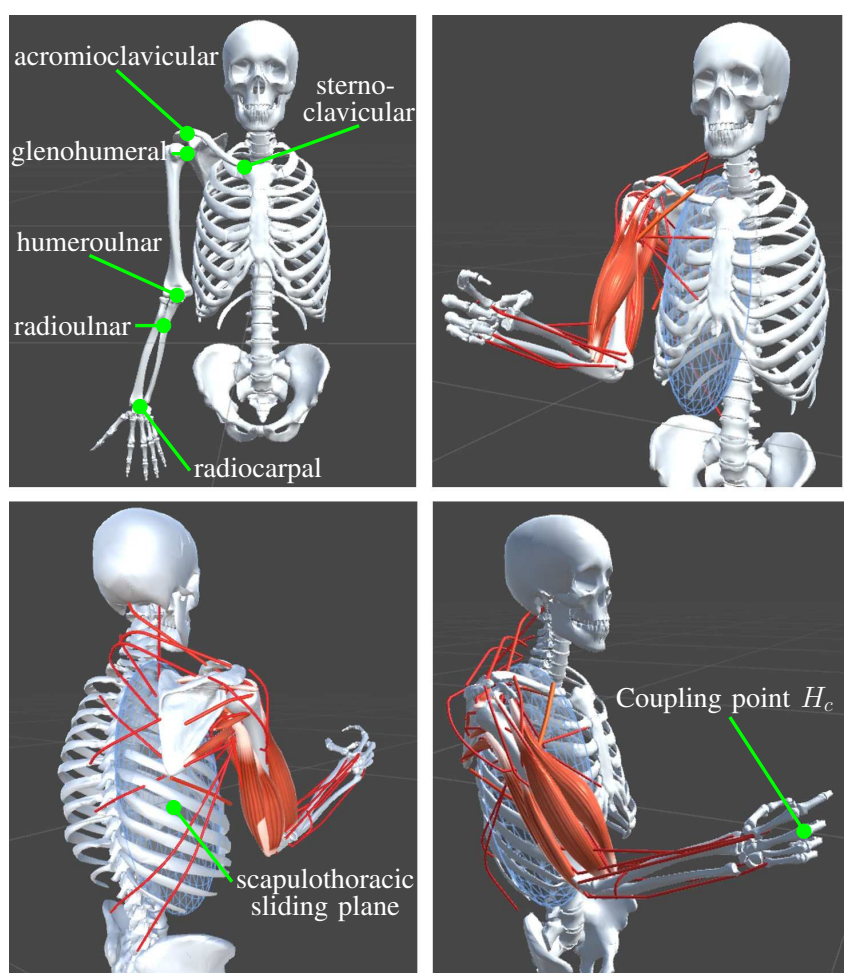

Fig. 3. Visualization of the 12 skeletal-DoF and 42 muscular-DoF human upper-limb musculoskeletal model in Unity. Several muscles such as musculi teres major et minor and musculi bi-et triceps brachii are implemented as a 3D model, whereas the other muscles are represented by red cables.

The verification and more details of this upper-limb musculoskeletal system will be left for a consecutive paper focusing on human upper-limb neuromechanics and control. For the current work, the model serves solely as plant dynamics for the human motor control hypothesis outlined next.

\section{B. Kinematic synergy identification approach}

In order to reduce the control variables for the latentspace controller, the synergy of the desired motion needs to be identified expressing the high-dimensional movement in fewer coordinates.

Starting from a demonstrated joint-space trajectory $\boldsymbol{q}_{\mathrm{H}, \mathrm{demo}}(t) \in \mathbb{R}^{j} \forall t$, kinematic synergies can e.g. be identified via Principle Component Analysis (PCA) [18] approximating the original motion by

$$
\begin{gathered}
\boldsymbol{q}_{\mathrm{H}, \mathrm{demo}}(t) \approx \boldsymbol{W} \boldsymbol{q}_{\mathrm{L}}(t)+\overline{\boldsymbol{q}}_{\mathrm{H}, \mathrm{demo}}, \\
\boldsymbol{W}^{\mathrm{T}} \boldsymbol{W}=\boldsymbol{I}_{s},
\end{gathered}
$$

where $\boldsymbol{W} \in \mathbb{R}^{j \times s}$ is the transformation matrix between the joint and latent space, $\overline{\boldsymbol{q}}_{\mathrm{H} \text {,demo }}=$ const. is the estimated average posture of $\boldsymbol{q}_{\mathrm{H} \text {,demo }}$ over time, $\boldsymbol{q}_{\mathrm{L}} \in \mathbb{R}^{s}$ the latentspace coordinates, $s$ the latent-space dimensionality, and $\boldsymbol{I}_{s} \in \mathbb{R}^{s \times s}$ the identity matrix. $\boldsymbol{W}$ is obtained by applying Singular Value Decomposition (SVD) on a data matrix representing the trajectory $\boldsymbol{q}_{\mathrm{H}, \mathrm{demo}}(t) \forall t$, see [13]. Note that $\boldsymbol{W}$ and $\overline{\boldsymbol{q}}_{\mathrm{H} \text {,demo }}$ are task-specific, i.e., for a different $\boldsymbol{q}_{\mathrm{H}, \mathrm{demo}}(t) \forall t$ (different task), a new $\boldsymbol{W}$ and $\overline{\boldsymbol{q}}_{\mathrm{H}, \mathrm{demo}}$ will be identified.

\section{Latent-space control hypothesis}

For the sole purpose of evaluating our framework, a potential first candidate for synergy-based upper limb control is introduced. However, note that its validity is not of interest at this stage. The human muscle-space controller is composed of 1) a latent-space trajectory controller, 2) a nullspace posture controller, and 3) a muscle-force controller, see Fig. 4.

The latent-space trajectory controller is defined as

$$
\begin{aligned}
\boldsymbol{F}_{\mathrm{M}, \mathrm{L}} & =\boldsymbol{K}_{\mathrm{M}, \mathrm{L}} \boldsymbol{J}_{\mathrm{M}} \boldsymbol{W}\left[\boldsymbol{q}_{\mathrm{L}, \mathrm{d}}-\boldsymbol{q}_{\mathrm{L}}\right] \\
& +\boldsymbol{D}_{\mathrm{M}, \mathrm{L}} \boldsymbol{J}_{\mathrm{M}} \boldsymbol{W}\left[\dot{\boldsymbol{q}}_{\mathrm{L}, \mathrm{d}}-\dot{\boldsymbol{q}}_{\mathrm{L}}\right],
\end{aligned}
$$

where $\boldsymbol{K}_{\mathrm{M}, \mathrm{L}}, \boldsymbol{D}_{\mathrm{M}, \mathrm{L}} \in \mathbb{R}^{42 \times 42}$ denote constant musclespace impedance matrices, and $\boldsymbol{q}_{\mathrm{L}, \mathrm{d}}$ the desired latent-space coordinates.

The posture controller intents to keep the system in the neighborhood of the average posture $\overline{\boldsymbol{q}}_{\mathrm{H} \text {,demo }}$. It is defined as

$$
\begin{array}{r}
\boldsymbol{F}_{\mathrm{M}, \mathrm{P}}=\boldsymbol{K}_{\mathrm{M}, \mathrm{P}} \boldsymbol{J}_{\mathrm{M}} \boldsymbol{N}_{\boldsymbol{W}^{\mathrm{T}}} \boldsymbol{N}_{\boldsymbol{W}^{\mathrm{T}}}^{\mathrm{T}}\left[\overline{\boldsymbol{q}}_{\mathrm{H}, \mathrm{demo}}-\boldsymbol{q}_{\mathrm{H}}\right] \\
-\boldsymbol{D}_{\mathrm{M}, \mathrm{P}} \boldsymbol{J}_{\mathrm{M}} \boldsymbol{N}_{\boldsymbol{W}^{\mathrm{T}}} \boldsymbol{N}_{\boldsymbol{W}^{\mathrm{T}}}^{\mathrm{T}} \dot{\boldsymbol{q}}_{\mathrm{H}},
\end{array}
$$

where $\boldsymbol{K}_{\mathrm{M}, \mathrm{P}}, \boldsymbol{D}_{\mathrm{M}, \mathrm{P}} \in \mathbb{R}^{42 \times 42}$ denote the muscle-space impedance matrices, and $\boldsymbol{N}_{\boldsymbol{W}^{\mathrm{T}}}$ an orthonormal basis for the null space of $\boldsymbol{W}^{\mathrm{T}}$. With the desired muscle-space force

$$
\boldsymbol{F}_{\mathrm{M}, \mathrm{d}}=\max \left(\mathbf{0}, \boldsymbol{F}_{\mathrm{M}, \mathrm{L}}+\boldsymbol{F}_{\mathrm{M}, \mathrm{P}}\right)
$$

the muscle-force controller outputs the muscle activation

$$
\boldsymbol{u}=\int \boldsymbol{T}^{-1} \boldsymbol{\Psi}^{-1}\left(\boldsymbol{q}_{\mathrm{H}}, \dot{\boldsymbol{q}}_{\mathrm{H}}\right)\left[\boldsymbol{F}_{\mathrm{M}, \mathrm{d}}-\boldsymbol{F}_{\mathrm{M}}\right]
$$

Herein $\boldsymbol{T} \in \mathbb{R}^{42 \times 42}$ denotes the positive diagonal integral time constant matrix, and $\boldsymbol{\Psi}\left(\boldsymbol{q}_{\mathrm{H}}, \dot{\boldsymbol{q}}_{\mathrm{H}}\right) \in \mathbb{R}^{42 \times 42}$ the diagonal matrix for muscle force-activation linearization, i.e.,

$$
\boldsymbol{\Psi} \approx \frac{\partial \boldsymbol{f}\left(\boldsymbol{u}, \boldsymbol{l}_{\mathrm{M}}\left(\boldsymbol{q}_{\mathrm{H}}\right), i_{\mathrm{M}}\left(\boldsymbol{q}_{\mathrm{H}}, \dot{\boldsymbol{q}}_{\mathrm{H}}\right)\right)}{\partial \boldsymbol{u}} .
$$

The musculotendious parameters, such as maximum isometric force or muscle optimum length, in function $f(\cdot)$ are taken from [19]. In future work, we will plan to extend the controller with muscular synergies (muscular latent space) and latent-space impedance matrices. 


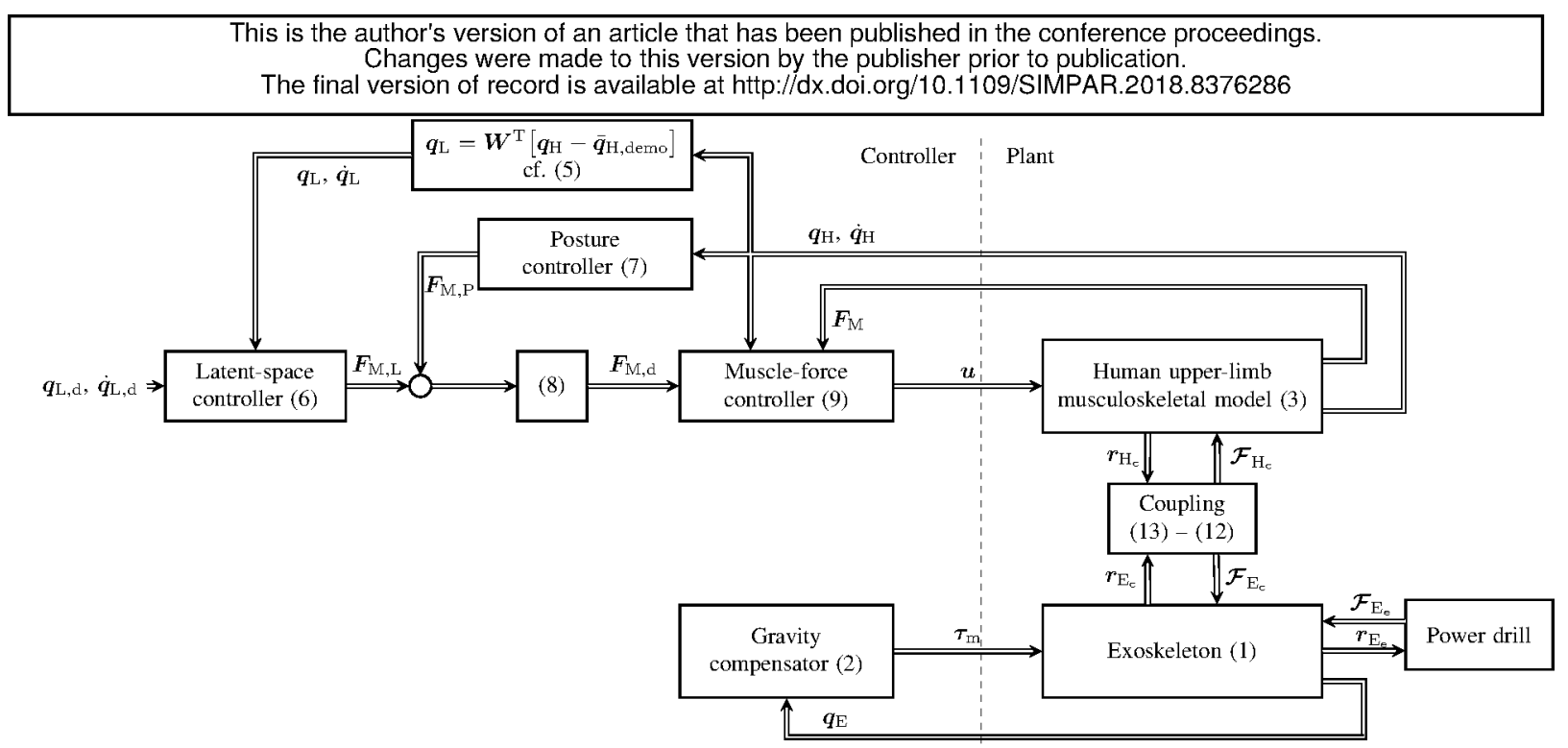

Fig. 4. Block diagram of the heterogeneous coupled human-exoskeleton system with human muscle-space control.

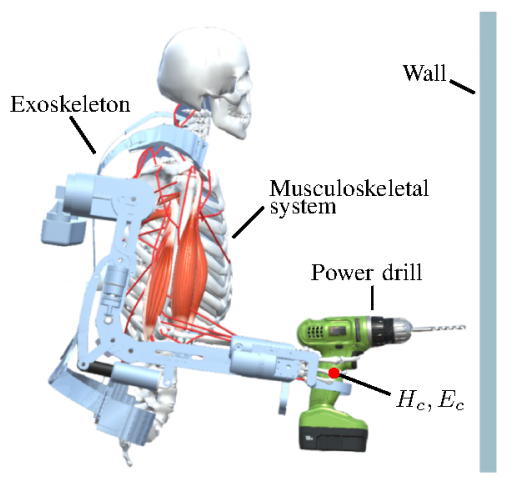

Fig. 5. Visualization of the human-exoskeleton system, where $H_{c}$ and $E_{c}$ denote the coupling points.

\section{Joint-space trajectory control}

For comparison with the proposed controller defined by $(6),(7)$ and (8), we use a basic joint-space trajectory controller, which is defined as

$$
\begin{aligned}
\boldsymbol{F}_{\mathrm{M}, \mathrm{d}}=\max & \left(\mathbf{0}, \boldsymbol{K}_{\mathrm{M}, \mathrm{J}} \boldsymbol{J}_{\mathrm{M}}\left[\boldsymbol{q}_{\mathrm{H}, \text { demo }}-\boldsymbol{q}_{\mathrm{H}}\right]\right. \\
& \left.+\boldsymbol{D}_{\mathrm{M}, \mathrm{J}} \boldsymbol{J}_{\mathrm{M}}\left[\dot{\boldsymbol{q}}_{\mathrm{H}, \text { demo }}-\dot{\boldsymbol{q}}_{\mathrm{H}}\right]\right),
\end{aligned}
$$

where $\boldsymbol{K}_{\mathrm{M}, \mathrm{J}}, \boldsymbol{D}_{\mathrm{M}, \mathrm{J}} \in \mathbb{R}^{42 \times 42}$ denote the diagonal musclespace impedance matrices.

\section{COUPLED HUMAN-EXOSKELETON SYSTEM}

For building a heterogenous human-exoskeleton simulation, the human upper-limb neuromechanics and the exoskeleton dynamics have to be coupled meaningfully, see Fig. 5.

For sake of simplicity, the subsystems are assumed to have a fixed base, meaning the human thorax and the exoskeleton shoulder bases are fixed in space and are not able to move. However, the $3 \mathrm{rd} \mathrm{Arm}$ is designed to be wearable, thus a floating base extension will be considered in the future.

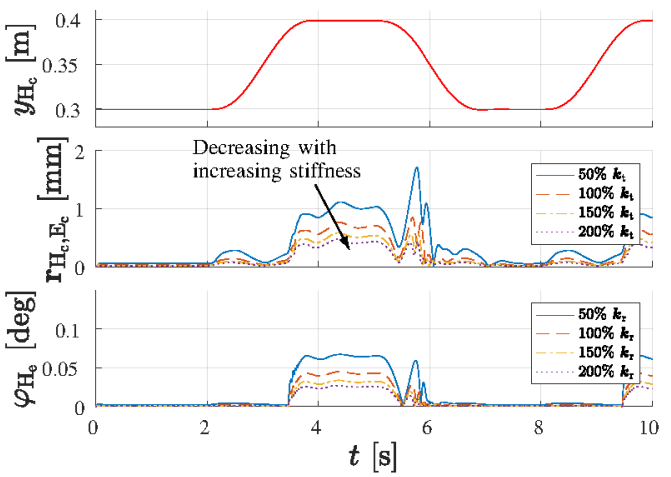

Fig. 6. Modeling of spring coupling by varying the reference spring stiffness $\boldsymbol{k}_{\mathrm{t}}=\left[\begin{array}{lll}5 & 5 & 5\end{array}\right]^{\mathrm{T}} \mathrm{kN} / \mathrm{m}$ and $\boldsymbol{k}_{\mathrm{r}}=\left[\begin{array}{llll}2.86 & 2.86 & 2.86\end{array}\right]^{\mathrm{T}} \mathrm{kNm} / \mathrm{deg}$, see (13) and (14). $y_{\mathrm{H}_{c}}$ denotes the Cartesian position of $H_{c}$ in $y$-direction expressed in world coordinates.

\section{A. Coupling stiffness}

On the exoskeleton side, coupling is assumed to act through the handle where also the power drill is attached, see $E_{c}$ in Fig. 2. On the human side, the coupling point is located at the human hand, see $H_{c}$ in Fig. 3. It is modeled as a 6-dimensional virtual spring according [20]. Physically, this 6-dimensional virtual spring is equivalent to three rotatory 1-DoF springs mounted in a gimbal structure connecting the hand and the handle together with three translational 1-DoF springs.

The wrenches acting at point $E_{c}$ and $H_{c}$ expressed in local spring frame are defined as

$$
\mathcal{F}_{\mathrm{H}_{\mathrm{c}}}=\left(\begin{array}{c}
\boldsymbol{f}_{\mathrm{H}_{\mathrm{c}}} \\
\boldsymbol{m}_{\mathrm{H}_{\mathrm{c}}}
\end{array}\right)=-\mathcal{F}_{\mathrm{E}_{\mathrm{c}}} .
$$

The resulting coupling force $\boldsymbol{f}_{\mathrm{H}_{\mathrm{c}}} \in \mathbb{R}^{3}$ is

$$
\boldsymbol{f}_{\mathrm{H}_{\mathrm{c}}}=-\operatorname{diag}\left(\boldsymbol{k}_{\mathrm{t}}\right) \boldsymbol{r}
$$

where $k_{\mathrm{t}} \in \mathbb{R}^{3}$ is the translational stiffness of the spring and $r \in \mathbb{R}^{3}$ the position difference between $H_{c}$ and $E_{c}$. The 


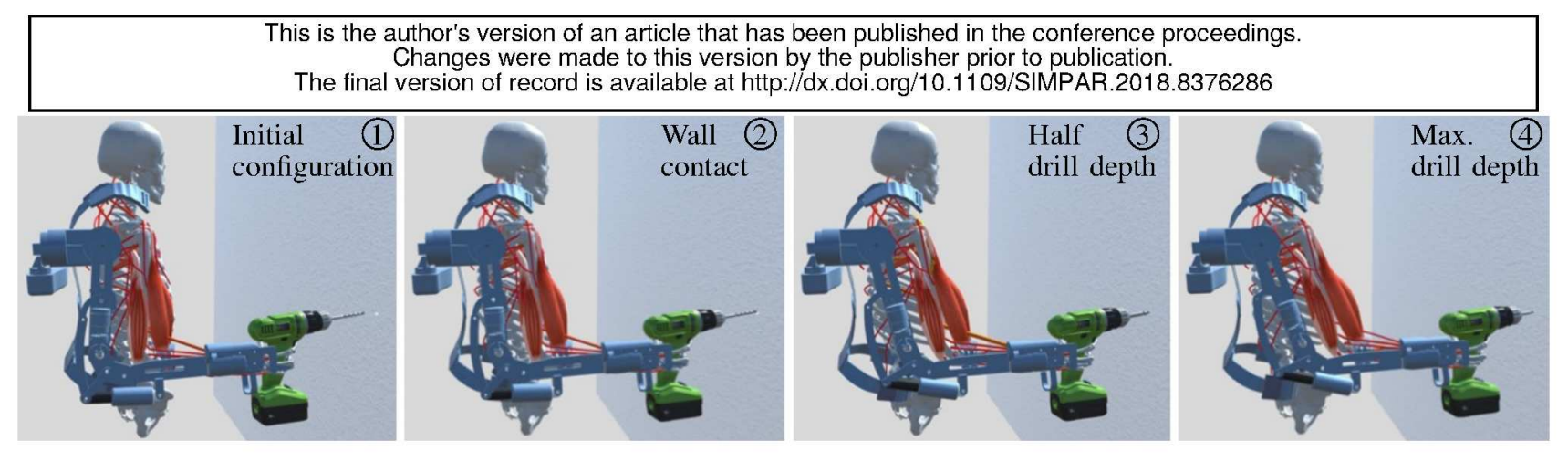

Fig. 7. Image sequence of the drilling task. See also the video attachment of this paper.

corresponding moment $m_{\mathrm{H}_{\mathrm{c}}} \in \mathbb{R}^{3}$ is

$$
\boldsymbol{m}_{\mathrm{H}_{\mathrm{c}}}=-\boldsymbol{J}_{\omega}^{-\mathrm{T}}(\boldsymbol{\varphi}) \operatorname{diag}\left(\boldsymbol{k}_{\mathrm{r}}\right) \boldsymbol{\varphi}
$$

where $\varphi \in \mathbb{R}^{3}$ is the orientation difference in Euler angle representation, $\boldsymbol{J}_{\omega} \in \mathbb{R}^{3 \times 3}$ is the Jacobian between Euler angle velocities and angular velocities and $\boldsymbol{k}_{\mathrm{r}} \in \mathbb{R}^{3}$ denotes the three rotational stiffness values, see [20].

This approach decouples the subsystems human and exoskeleton via a 6-DoF spring. The effect of the spring on the coupling behavior is shown for various stiffnesses in Fig. 6 during a Cartesian movement in $y$-direction, see Fig. 3. Obviously, the larger the stiffness the smaller the difference between $H_{c}$ and $E_{c}$. Additionally, a damper is placed in parallel to the spring in order to reduce unrealistic vibration effects.

\section{B. Alternative coupling approaches}

Other alternatives for coupling the subsystems would be to formulate implicit kinematic constraints resulting from the kinematic chain of both subsystems. In this case, one could calculate the constraint forces via Lagrange multipliers and the coordinate partitioning approach [21], or via Gauss's principle of least constraints via Udwadia-Kalaba equation [22]. However, these methods are more complex and computationally costly. Furthermore, the proposed coupling spring approach exhibits a reasonable pose difference during operation, see Fig. 6.

\section{Process Model}

In this paper, the power drilling process is modeled as a time-varying process wrench $\mathcal{F}_{\mathrm{p}}(t) \in \mathbb{R}^{6}$ described in task frame. It is composed of the Cartesian forces and moments, acting at the endeffector of the exoskeleton, i.e., $\mathcal{F}_{\mathrm{E}_{\mathrm{c}}}=$ $\boldsymbol{R} \mathcal{F}_{\mathrm{p}}$ in (1). $\boldsymbol{R} \in \mathbb{R}^{6 \times 6}$ denotes the wrench rotation matrix from task to inertial frame.

\section{SIMULATION RESULTS}

In the overall evaluation scenario, a human craftsman is equipped with an exoskeleton drilling a hole into a wall using a power drilling machine, see Fig. 7. The human is controlled in muscle space following the latent trajectory of an exemplary drilling movement. The exoskeleton compensates for gravity effects of the power tool. Furthermore, a time-varying process force $\mathcal{F}_{P}$ is applied when contacting the wall. In this paper, following investigations are made.

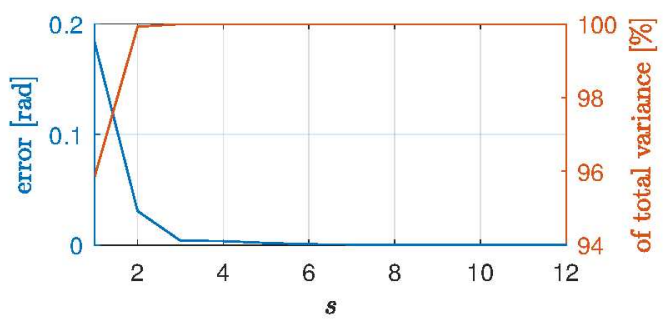

Fig. 8. PCA identification of the kinematic latent space. An s-dimensional latent representation describes \% of total variance with a certain reconstruction error.

1) We evaluate the tracking behavior when the controller follows a low-dimensional trajectory (Sec. III-C) or a high-dimensional trajectory (Sec. III-D). We would expect essentially the same tracking accuracy while considering fewer dimensions.

2) We analyze human muscle effort $\boldsymbol{\xi}$ (see (16)) with and without exoskeletal assistance. In the former case, we expect significantly reduced muscle effort. Furthermore, we analyze $\boldsymbol{\xi}$ during external wrenches $\mathcal{F}_{P} \neq \mathbf{0}$ in comparison to a contact-free movement $\mathcal{F}_{P}=0$, where a reduced muscle effort $\boldsymbol{\xi}$ would be expected.

\section{A. Kinematic synergy identification}

Based on a given 12-DoF human joint-space drilling trajectory $\boldsymbol{q}_{\mathrm{H}, \mathrm{demo}}{ }^{3}$, a two-dimensional latent-space trajectory $\boldsymbol{q}_{\mathrm{L}}$ is identified (see Sec. III-B). Fig. 8 shows the results of PCA, leading to $s=2$, cf. (5). In the following, the identified latent-space is used to generate the input for the human muscle-space controller from Sec. III-C.

\section{B. Latent-space trajectory vs. joint-space trajectory control}

The human muscle-space controller parameters in (6), (7) and (9) are determined empirically to achieve satisfactory tracking behavior: $\boldsymbol{K}_{\mathrm{M}, \mathrm{L}}=\boldsymbol{K}_{\mathrm{M}, \mathrm{P}}=4 \mathrm{MN} / \mathrm{m} \boldsymbol{I}_{42}, \boldsymbol{D}_{\mathrm{M}, \mathrm{L}}=$ $\boldsymbol{D}_{\mathrm{M}, \mathrm{P}}=60 \mathrm{kNs} / \mathrm{m} \boldsymbol{I}_{42}$ and $\boldsymbol{T}^{-1}=100 \mathrm{~s}^{-1} \boldsymbol{I}_{42}$. The process wrench is set to

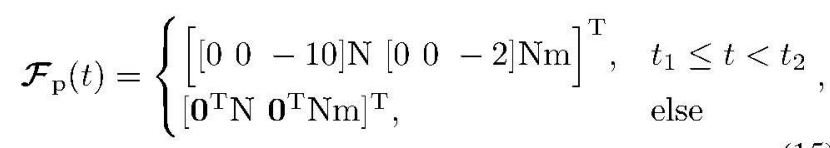

\footnotetext{
${ }^{3}$ This joint-space trajectory is generated by applying inverse kinematics to a predefined Cartesian path.
} 


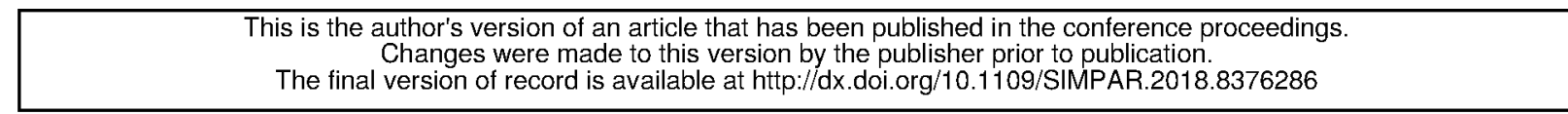

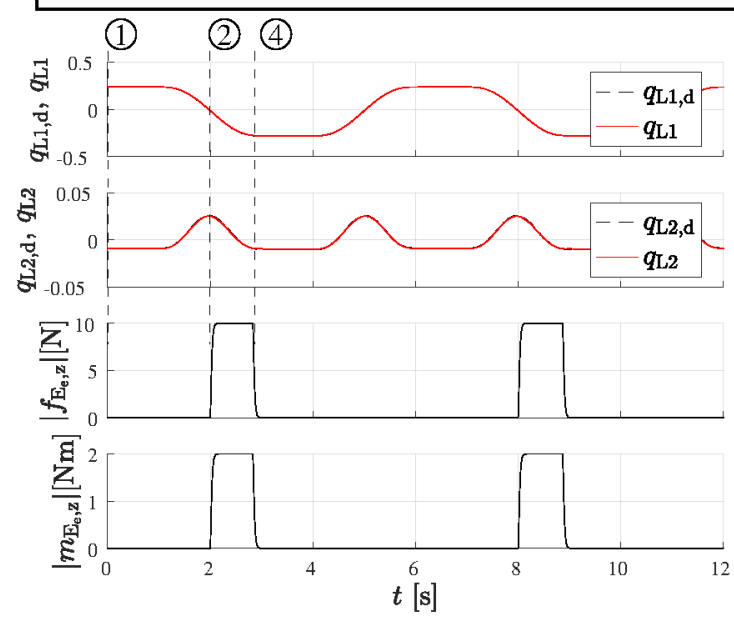

Fig. 9. Tracking of latent coordinates $q_{\mathrm{L}}$ during two consecutive cycles of a drilling task. The annotated numbers 1,2 and 4 correspond to Fig. 7.

where $t_{1}$ is the time of wall contact and $t_{2}$ is the time of reaching the maximum drilling depth. Note that the task and inertial frame remain parallel to each other throughout the entire drilling task. Fig. 9 shows good tracking performance for $\boldsymbol{q}_{\mathrm{L}}$ together with the applied process wrench $\mathcal{F}_{p}$ (see (15)).

Now, we compare this controller with the joint-space trajectory controller (11), when using the same controller parameters, i.e., $\boldsymbol{K}_{\mathrm{M}, \mathrm{J}}=\boldsymbol{K}_{\mathrm{M}, \mathrm{L}}=\boldsymbol{K}_{\mathrm{M}, \mathrm{P}}, \boldsymbol{D}_{\mathrm{M}, \mathrm{J}}=\boldsymbol{D}_{\mathrm{M}, \mathrm{L}}=$ $\boldsymbol{D}_{\mathrm{M}, \mathrm{P}}$. This means that instead of tracking only a twodimensional latent-space trajectory, this controller tracks all 12 original joints from $\boldsymbol{q}_{\mathrm{H}, \mathrm{demo}}$, resulting in $\boldsymbol{q}_{\mathrm{H}, \mathrm{J}}$.

Fig. 11 depicts the 12-dimensional joint positions of the originally demonstrated movement $q_{\mathrm{H} \text {,demo }}$ together with the results from the joint-space trajectory controller and the latent-space trajectory controller. As anticipated, the joint position error is nearly the same for both controllers. However, the latent-space trajectory requires fewer dimensions, while achieving similar tracking behavior.

\section{Muscle effort analysis}

Using the overall simulation framework one is able to investigate human muscle activities/efforts as well. For example, we evaluate the effectiveness of the exoskeletal assistance. As an evaluation metric we define muscle effort $\xi \in \mathbb{R}^{42}$ to be

$$
\boldsymbol{\xi}=\int_{0}^{t_{\mathrm{end}}} \boldsymbol{u}(t) \mathrm{d} t
$$

where $t_{\text {end }}$ denotes the end of the drilling task. As one expects, Fig. 10 shows that the muscle efforts are significantly reduced when the human user is assisted by the exoskeleton. Furthermore, we compare the scenarios with and without the process wrench given by (15). Clearly, only slightly smaller $\boldsymbol{\xi}$ is required.

\section{CONCLUSION}

In this work, we developed a more complete and complex dynamics simulation tool for an upper-limb humanexoskeleton system performing a exemplary power drilling

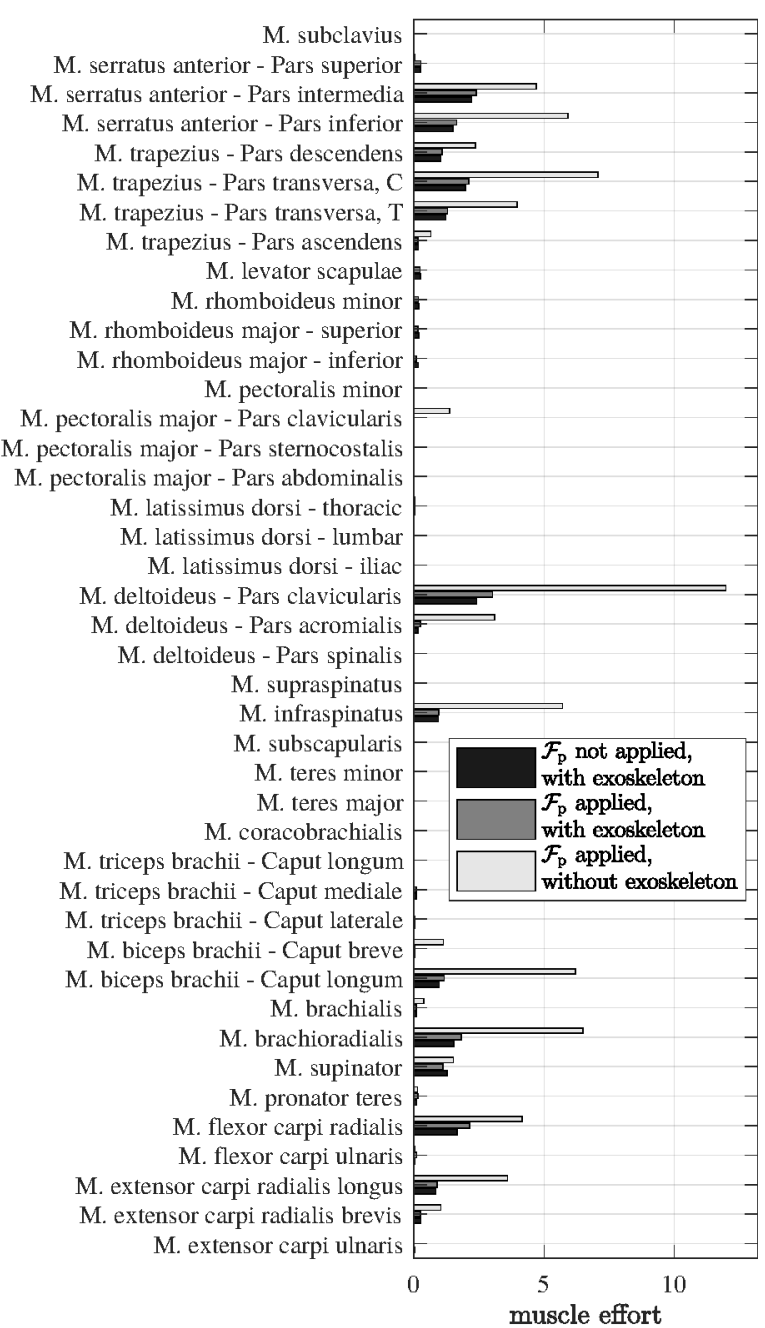

Fig. 10. The muscle effort exerted by each muscle for the power drilling task with and without the process force $\mathcal{F}_{\mathrm{p}}$, as well as with and without exoskeletal assistance.

task. With this, it is possible to design and evaluate diverse human motor control hypotheses in different manipulation tasks. Besides, one may investigate human-centered exoskeleton controllers regarding human and exoskeleton posture, tracking error, muscle activation/effort, or interaction wrenches. Exemplarily, we implemented and evaluated a human motor latent-space trajectory control hypothesis, which provides a similar tracking behavior as a standard joint-space trajectory controller. Furthermore, we analyzed human muscle effort 1) by comparing the scenarios with and without exoskeletal assistance and 2) for an occurring external wrench compared to a contactless movement.

Building on this tool, future work will address

1) the extension to a floating base controlled exoskeleton,

2) the extension of the human musculoskeletal model with more muscles and further limbs,

3) the enhancement of the proposed human motor control algorithm with muscle synergies and latent-space 

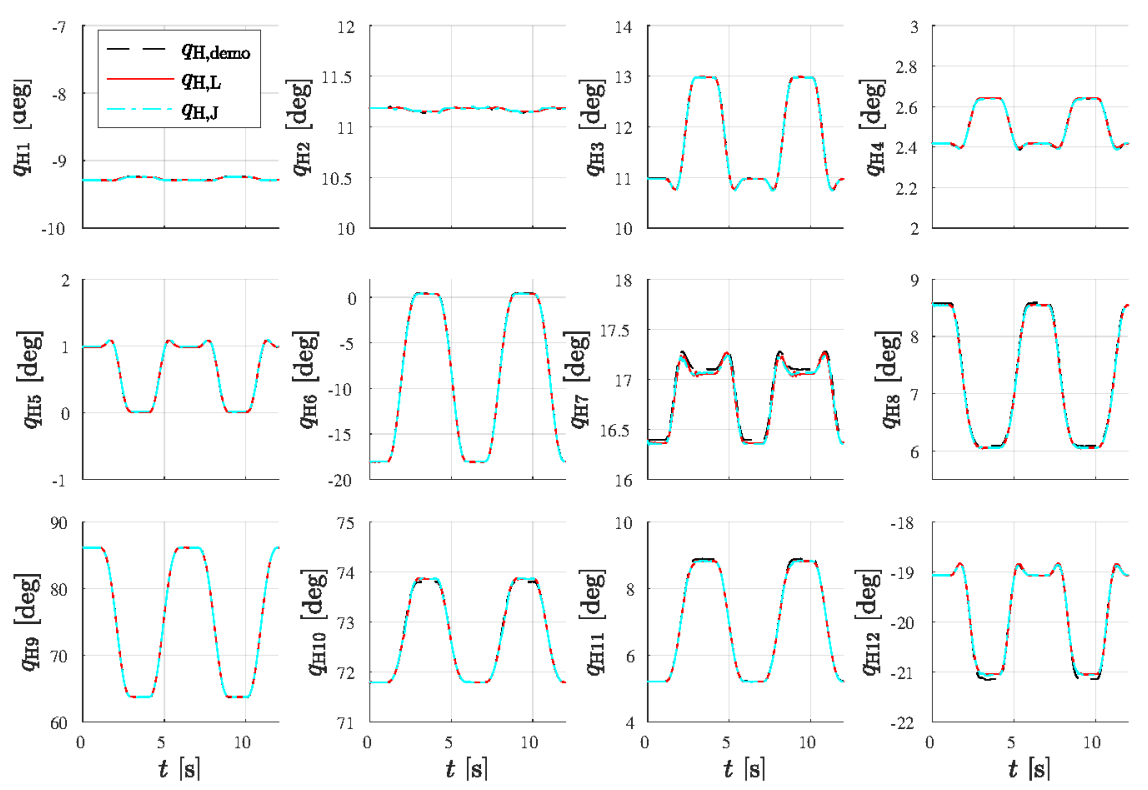

Fig. 11. Human joint angles $\boldsymbol{q}_{\mathrm{H}}$ resulting from latent-space trajectory control $\boldsymbol{q}_{\mathrm{H}, \mathrm{L}}$, joint-space trajectory control $\boldsymbol{q}_{\mathrm{H}, \mathrm{J}}$ and original joint angles $\boldsymbol{q}_{\mathrm{H}, \text { demo }}$.

impedance, including stability analysis,

4) task-specific and human-centered exoskeleton control that helps execute tasks, and

5) the implementation of a more realistic process model.

\section{ACKNOWLEDGMENT}

We greatly acknowledge the funding of this work by the Alfried Krupp von Bohlen und Halbach Foundation, the European Unions Horizon 2020 grant "SOFTPRO" (No. 688857), and the German Federal Ministry of Education and Research grant "3rd Arm" (No. 16SV6175).

\section{REFERENCES}

[1] M. Casadio, V. Sanguineti, P. Morasso, and V. Arrichiello, "Braccio di Ferro: A new haptic workstation for neuro-motor rehabilitation," Technology and Health Care, vol. 14, p. 123142, 2006.

[2] T. Nef, M. Mihelj, and R. Riener, "ARMin: a robot for patientcooperative arm therapy," Medical \& Biological Engineering \& Computing, vol. 45, no. 9, pp. 887-900, 2007.

[3] J. Rosen, M. Brand, M. B. Fuchs, and M. Arcan, "A myosignal-based powered exoskeleton system," IEEE Trans. Syst. Man Cybern. A Syst. Hum., vol. 31, no. 3, pp. 210-222, 2001.

[4] A. Chu, H. Kazerooni, and A. Zoss, "On the Biomimetic Design of the Berkeley Lower Extremity Exoskeleton (BLEEX)," in Proc. of the IEEE Int. Conf. on Robotics and Automation (ICRA), 2005, p. 43564363.

[5] H.-T. Tran, H. Cheng, M.-K. Duong, and H. Zheng, "Fuzzy-based impedance regulation for control of the coupled human-exoskeleton system," in IEEE ROBIO, Bali, Indonesia, December 2014.

[6] D. G. Thelen, F. C. Anderson, and S. L. Delp, "Generating dynamic simulation of movement using computed muscle control," J. Biomech., vol. 36 , pp. 321-328, 2003.

[7] D. G. Thelen and F. C. Anderson, "Using computed muscle control to generate forward dynamic simulation of human walking from experimental data," J. Biomech., vol. 39, pp. 1107-1115, 2006.

[8] S.-H. Lee and D. Terzopoulos, "Heads up! Biomechanical modeling and neuromuscular control of the neck," in ACM SIGGRAPH Conf., Boston, MA, August 2006.

[9] R. S. Razavian, N. Mehrabi, and J. McPhee, "A model-based approach to predict muscle synergies using optimization: application to feedback control," Front. Comput. Neurosci., vol. 9, pp. 1-13, 2015.
[10] M. C. Tresch and A. Jarc, "The case for and against muscle synergies," Current Opinion in Neurobiology, vol. 19, no. 6, pp. 601-607, Dec. 2009.

[11] M. Santello, M. Flanders, and J. F. Soechting, "Postural hand synergies for tool use," Journal of Neuroscience, vol. 18, no. 23, 1998.

[12] M. Santello and C. E. Lang, "Are movement disorders and sensorimotor injuries pathologic synergies? when normal multi-joint movement synergies become pathologic," Frontiers Human Neuroscience, vol. 8, 2014.

[13] T. Wimböck, B. Jahn, and G. Hirzinger, "Synergy level impedance control for multifingered hands," in IROS, San Francisco, CA, USA, September 2011.

[14] D. Pan, F. Gao, Y. Miao, and R. Cao, "Co-simulation research of a novel exoskeleton-human robot system on humanoid gaits with fuzzyPID/PID algorithms," Adv. Eng. Softw., vol. 79, pp. 36-46, 2015.

[15] A. F. Ruiz, E. Rocon, R. Raya, and J. L. Pons, "Coupled control of human-exoskeleton systems: an adaptive process," in Conf. HSI, Krakow, Poland, 2008.

[16] M. Azad and Featherstone, "Modeling the contact between a rolling sphere and a compliant ground plane," in ACRA, 2010, pp. 1-7.

[17] J. M. Winters, "Hill-based muscle models: a systems engineering perspective," in Multiple muscle systems: biomechanics and movement organization, J. M. Winters and S. L. Woo, Eds. Springer-Verlag, 1990 , ch. 5, pp. 69-93.

[18] I. T. Jolliffe, Principal Component Analysis, 2nd ed. Springer-Verlag New York, Inc., 2002.

[19] B. A. Garner and M. G. Pandy, "Musculoskeletal model of the upper limb based on the visible human male dataset," Comput. Meihods Biomech. Biomed. Engin., vol. 4, pp. 93-126, 2001.

[20] C. Natale, Interaction Control of Robot Manipulators: Six-Degrees-ofFreedom Tasks. Springer, 2003, vol. 3.

[21] R. Wehage and E. Haug, "Generalized coordinate partitioning for dimension reduction in analysis of constrained dynamic systems," $J$. Mech. Des., vol. 104, no. 1, pp. 247-255, 1982.

[22] F. E. Udwadia and R. E. Kalaba, "A new perspective on constrained motion," in Proceedings: Mathematical and Physical Sciences. JSTOR, 1992, pp. 407-410. 